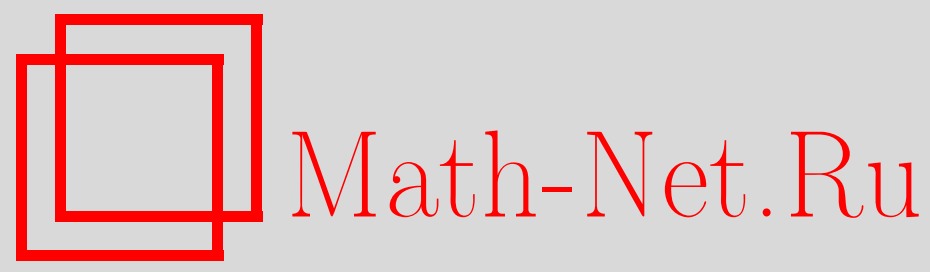

С. М. Агеев, С. А. Богатый, О препятствиях к продолжению частичных отображений, Матем. заметки, 1997, том 62, выпуск 6, 803-812

DOI: https://doi.org/10.4213/mzm1669

Использование Общероссийского математического портала Math-Net.Ru подразумевает, что вы прочитали и согласны с пользовательским соглашением http://www . mathnet.ru/rus/agreement

Параметры загрузки:

IP: 3.89 .185 .249

26 апреля 2023 г., $16: 24: 38$

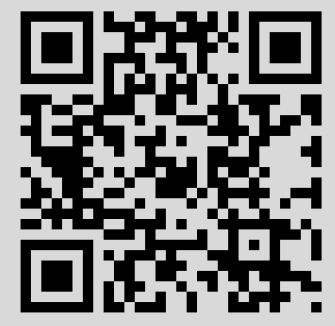




\title{
О ПРЕПЯТСТВИЯХ К ПРОДОЛЖЕНИЮ ЧАСТИЧНЫХ ОТОБРАЖЕНИЙ
}

\author{
С. М. Агеев, С. А. Богатый
}

Одной из важнейших задач топологии является минимизация (в том или ином смысле) препятствия к продолжению частичного отображения $Z \hookleftarrow A \stackrel{f}{\rightarrow} X$, т.е. такого подмножества $F \subset Z \backslash A$, на дополнении к которому существует глобалное продолжение $f$. Показано, что если фиксировать метрическое пространство $Z, \operatorname{dim} Z \leqslant n$, и числа $p, q \geqslant-1$, то препятствия ко всем частичньм отображениям $Z \hookleftarrow A \stackrel{f}{\rightarrow} X \in$ $\mathrm{LC}^{p} \cap \mathrm{C}^{q}$ можно сосредоточить в наперед выбранных достаточно тощих подмножествах $Z$.

Библиография: 12 названий.

1. Введение. Пусть на замкнутом подмножестве $A$ метрического пространства $Z$ задано отображение $f: A \rightarrow X$ в метрическое пространство $X$. Подмножество $F \subset Z \backslash A$ назьвается препятствием $к$ продолжснию частичного отображсения $Z \hookleftarrow A \stackrel{f}{\rightarrow} X$, если существует продолжение $\widehat{f}: Z \backslash F \rightarrow X, \widehat{f} \uparrow A=f$, отображения $f$ на $Z \backslash F$. Одной из важнейших задач топологии является минимизация (в том или ином смысле) препятствия к продолжению $f$. Так классическая теорема Куратовского-Дугунджи [1] утверждает, что $\operatorname{dim} F=-1$ (т.е. препятствие пусто), если $\operatorname{dim}(Z \backslash A) \leqslant n$, а $X$ является связным и локально связным в размерности $n-1\left(\right.$ кратко $\left.X \in \mathrm{C}^{n-1} \cap \mathrm{LC}^{n-1}\right)$. Определение связности и локальной связности в данной размерности можно найти в любом учебнике по теории ретрактов и гомотопий, например, в [2]. Если же потребовать от $X$ связности в меньшей размерности $q$ (т.е. $X \in \mathrm{C}^{q} \cap \mathrm{LC}^{n-1}$ ), то препятствие становится, вообще говоря, неустранимым. Однако, его всегда можно выбрать замкнутым в $Z$ и имеющим размерность не более $n-q-2$ (теорема Эйленберга-Борсука [3], [4]). При одновременном уменьшении связности и локальной связности $X, X \in \mathrm{C}^{q} \cap \mathrm{LC}^{p}$, где $q, p<n-1$, появляется еще одна компонента препятствия $F=F_{1} \cup F_{2}$, причем $F_{1}$ замкнуто в $Z \backslash A, F_{2}$ замкнуто в $Z, \operatorname{adim} F_{1} \leqslant n-p-2, \operatorname{dim} F_{2} \leqslant n-q-2$ (теорема Богатого [5]). Если в этой теореме $p=q=-1$ (т.е. $X$ - произвольное метрическое пространство), то препятствие для продолжения частичного отображения имеет размерность меньшую $n$ и замкнуто лежит в $Z \backslash A$ (теорема Левшенко [6]). Имеются результаты [7] о препятствиях к продолжению сечений многозначных полунепрерьвных снизу отображений.

Работа первого автора выполнена при частичной финансовой поддержке Международного научного фонда, грант NFU000, и Российского фонда фундаментальных исследований, грант № 93-011-1402. 
Большой интерес представляет вопрос о существовании замкнутых ретракций пространства $Z$. Р. Энгелькинг [8] положительно ответил на него для нульмерных метрических пространств $Z$ и их замкнутых подпространств $A \subset Z$. В работе [5] теоремы Энгелькинга и Левшенко были объединены: любое частичное замкнутое отображение $f$ имеет замкнутое в $Z \backslash A(\operatorname{dim} Z-1)$-мерное препятствие $F \subset Z \backslash A$ для продолжения его до замкнутого отображения.

Оказьвается, что приведенные выше теоремы, а также некоторые другие, допускают существенное уточнение. Так, например, если фиксировать метрическое пространство $Z, \operatorname{dim} Z \leqslant n$, и числа $p, q \geqslant-1$, то препятствие ко всем частичньм отображениям $Z \hookleftarrow A \stackrel{\varphi}{\rightarrow} X \in \mathrm{LC}^{p} \cap \mathrm{C}^{q}$ можно сосредоточить в наперед выбранных достаточно тощих подмножествах $Z$. Для точной формулировки этого и других доказываемых в статье фактов зафиксируем замкнутую и не более чем $(n+1)$-кратную сюръекцию $\eta: W \rightarrow Z$ из нульмерного метрического пространства $W$ на $Z$ (называемую резольвентой Мориты [9]) и построим возрастающую цепочку $F_{\sigma}$-множеств $\Phi_{n+2} \subset \Phi_{n+1} \subset \cdots \subset \Phi_{1}=Z$, $\operatorname{dim} \Phi_{k}=n+1-k$ (назьваемую фильтрацией, порожденной резольвентой Мориты, или просто фильтрацией Мориты):

$$
\Phi_{p}=\left\{z \in Z|| \eta^{-1}(x) \mid \geqslant p\right\}
$$

Все описанные вьше свойства этой фильтрации будут доказаны в предложении 6.

Теорема 1. Для любого частичного отобрахсения $Z \hookleftarrow A \stackrel{\varphi}{\rightarrow} X n$-мерного метрического пространства $Z$ в метрическое $\left(\mathrm{LC}^{p} \cap \mathrm{C}^{q}\right)$-пространство $X$, где $p$ и меньше $n$, существуют два мнохества $F_{1} \subset \Phi_{p+3}, F_{2} \subset \Phi_{q+3}$, лехащих в $Z \backslash A$, первое из которых замкнуто в $Z \backslash A$, а второе замкнуто в $Z$, таких, что отображсние $\varphi$ может быть продолжено в дополнение к ним, т.е. в $Z \backslash\left(F_{1} \cup F_{2}\right)$.

Отметим, что теорема 1 имеет доказательство значительно более простое и наглядное, как нам кажется, по сравнению с оригинальными доказательствами теорем Эйленберга-Борсука и Богатого. Существенно новым моментом является использование резольвенты Мориты не только в формулировке теоремы, но и в ее доказательстве.

Известно [2], что близкие отображения $n$-мерного метрического пространства $Y$ в $\mathrm{LC}^{n}$-пространство $X$ соединяются достаточно мелкой гомотопией. Следующая теорема учитывает ухудшение связностных свойств пространства $X$, из-за чего появляется неустранимое препятствие $F$, лежащее в произведении $Y \times(0,1)$.

Теорема 2. Пусть заданы метрическое $\mathrm{LC}^{p}$-пространство $X, p \leqslant n, u$ метрическое $n$-мерное пространство $Z=Y \times[0,1]$. Тогда для любого открытого покрытия $\alpha \in \operatorname{cov} X$ существует открытое покрытие $\beta \in \operatorname{cov} X$ такое, что для любых $\beta$-близких отображений $f, g: Y \rightarrow X$ существует замкнутое в $Y \times(0,1)$ множество $F \subset \Phi_{p+3}$, а также отображение $H: Z \backslash F \rightarrow X$ (называемое “дырявой" гомотопией $)$, соединяюшее $f u g, H|(Y \times\{0\})=f, H|(Y \times\{1\})=g, u$ ограниченное покрытием $\alpha$, т.е.

$$
\{H((y \times[0,1]) \backslash F) \mid y \in Y\} \succ \alpha .
$$

Теорема 3 гарантирует, что все препятствия к продолжению частичных замкнутых отображений до замкнутых отображений лежат в элементе $\Phi_{2}$ фильтрации Мориты. 
TЕоремА 3. Пусть $Z \hookleftarrow A \stackrel{\varphi}{\rightarrow} X$ есть частичное замкнутое отобрахсение $n$-мерного метрического пространства $Z$ в метрическое пространство $X$. Тогда существует замкнутое в $Z \backslash A$ множсество $F \subset \Phi_{2}$ такое, что $\varphi$ мохет бить продолжено до замкнутого отображения $\varphi: Z \backslash F \rightarrow X$.

Классическая конечномерная теорема Е. Майкла [10], [11] о селекции имеет дело с продолжением частичной селекции (селекцией многозначного отображения $\varphi$ называется однозначное непрерьвное отображение $s$, для которого $s(a) \in \varphi(a)$ для всех $a \in A)$ $Z \hookleftarrow A \stackrel{s}{\rightarrow} X$ многозначного полунепрерывного отображения $\varphi: Z \rightarrow X$, у которого $\varphi(z) \in \mathrm{LC}^{n-1} \cap \mathrm{C}^{n-1}$, а семейство $\{\varphi(z) \mid z \in Z\}$ замкнутых подмножеств в $X$ равностепенно локально связно в размерностях, не превосходящих $n-1$ (краткое обозначение: $\{\varphi(z) \mid z \in Z\} \in$ equi - $\left.\mathrm{LC}^{n-1}\right)$. В работе [7] были проанализированы последствия ухудшения связностных свойств $X$. В частности, было доказано, что если $Z$ есть метрическое $n$-мерное пространство, а $\varphi(z) \in \mathrm{LC}^{n-1} \cap \mathrm{C}^{q},\{\varphi(z) \mid z \in Z\} \in$ equi - $\mathrm{LC}^{n-1}$, то появляется замкнутое препятствие $F, \operatorname{dim} F \leqslant n-q-2$, к продолжению сечения $s$. В теореме 4 утверждается, что все препятствия могут быть сосредоточены в элементе $\Phi_{q+3}$ фильтрации Мориты.

Теорема 4. Пусть $(Z, d)$ есть метрическое $n$-мерное пространство, $\varphi: Z \rightarrow X$ есть полнозначное полунепрерывное снизу многозначное отображсние, для которого $\varphi(z) \in \mathrm{LC}^{n-1} \cap \mathrm{C}^{q}$ для всех $z \in Z$, а семейство $\{\varphi(z) \mid z \in Z\} \in$ equi- $\mathrm{LC}^{n-1}$. Тогда для любой частичной селекиии $Z \hookleftarrow A \stackrel{s}{\rightarrow} X$ отображсения $\varphi$ существуют замкнутое в $Z$ мнохсество $F \subset \Phi_{q+3} \backslash A$ и селекиия $\widehat{s}: Z \backslash F \rightarrow X$, являющаяся продолэением селекиии $s$.

Доказательная часть статьи будет построена следующим образом. После введения предварительных понятий и сведений будет дано подробное доказательство теоремы 1. Из-за ограниченности объема работы доказательства оставшихся теорем не будут приводиться. Однако, заинтересованньй читатель найдет здесь все необходимое для их восстановления.

Недавно А. Н. Дранишников [12] получил весьма важное обобщение теоремы Эйленберга-Борсука. С ее помощью ему удалось найти эффектные решения нескольких проблем гомологической теории. Напомним, что для сепарабельных метрических пространств $\operatorname{dim} Z \leqslant n$ тогда и только тогда, когда $Z \in \mathrm{AE}\left(S^{n}\right)$ (т.е. любое частичное отображение $Z \hookleftarrow A \stackrel{f}{\rightarrow} S^{n}$ имеет глобальное продолжение $\left.f: Z \rightarrow S^{n}\right)$. Воспользовавшись также совпадением сферы $S^{n+m+1}$ и джойна $S^{n} * S^{m}$ сфер $S^{n}$ и $S^{m}$, частньй случай теоремы Эйленберга-Борсука $\left(X=S^{n}\right)$ можно переформулировать так:

если $Z \in \mathrm{AE}\left(S^{n} * S^{m}\right)$, то для любого частичного отображения $Z \hookleftarrow A \stackrel{f}{\rightarrow} S^{n}$ имеется препятствие $F \subset Z \backslash A, F \in \mathrm{AE}\left(S^{m}\right)$.

Обобщение Дранишникова заключается в замене сфер $S^{n}$ и $S^{m}$ на произвольные счетные $\mathrm{CW}$-комплексы $K$ и $L$ соответственно:

если $Z \in \mathrm{AE}(K * L)$, то для любого частичного отображения $Z \hookleftarrow A \stackrel{f}{\rightarrow} K$ имеется препятствие $F \subset Z \backslash A, F \in \mathrm{AE}(L)$.

Вопрос. Верно ли, что все препятствия $F$ мохно сосредоточить в некотором множестве $\Phi \in \mathrm{AE}(L)$ ? Точнее, если $Z \in \mathrm{AE}(K * L)$, то существует ли такое множество $\Phi \subset Z, \Phi \in \mathrm{AE}(L)$, что для любого частичного отображсения $Z \hookleftarrow A \stackrel{f}{\rightarrow} K$ имеется препятствие $F \subset(Z \backslash A) \cap \Phi, F \in \mathrm{AE}(L) ?$ 
2. Предварительные понятия и сведения. В теории продолжений частичных отображений большую роль играют критерии абсолютньх окрестностных экстензоров, основьвающиеся на понятии реализации полиэдров (условия Лефшеца). И в нашем изложении модифицированньй критерий Лефшеца также будет играть первостепенную роль, позволяя избегать достаточно обременительной конструкции присоединенного пространства. Мы позволим себе назвать вводимьй ниже в предложении 2 принцип продолжения отображений именем Лефшеца.

ОПРЕДЕЛЕНИЕ 1. Пусть $\alpha$ есть система открытых множеств пространства $X$ (под пространствами мы будем понимать только метрические пространства, под отображениями - только непрерьвные отображения), $\mathfrak{N}_{0}$ есть подполиэдр полиэдра $\mathfrak{N}$, содержащий все его вершины. Частичной $\alpha$-реализацией полиэдра $\mathfrak{N}$ (или $\alpha$-реализацией)

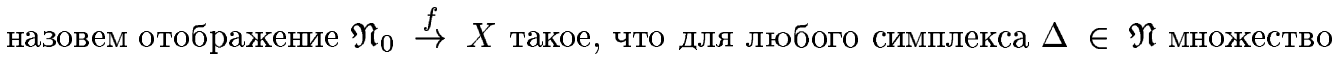
$f\left(\Delta \cap \mathfrak{N}_{0}\right)$ содержится в некотором элементе $V \in \alpha$.

ОПРЕДЕЛЕНИЕ 2. Пространство $X$ обладает свойством Лефиеца $\operatorname{Lf}^{p}, p \leqslant \infty$, если для любого покрытия $\delta \in \operatorname{cov} X$ (под покрытием пространств мы будем понимать открытые, необязательно локально конечные, покрытия; все основные понятия, связанные с покрытиями, можно найти, например, в [9]) существует покрытие $\gamma \in \operatorname{cov} X$ такое, что для любой частичной $\gamma$-реализации $\mathfrak{N} \hookleftarrow \mathfrak{N}_{0} \stackrel{f}{\rightarrow} X$ полиэдра $\mathfrak{N}$ размерности не больше $p+1$ существует $\delta$-реализация $\widehat{f}: \mathfrak{N} \rightarrow X$, продолжающая $f, \widehat{f} \mid \mathfrak{N}_{0}=f$.

Зависимость $\gamma$ от $\delta$ будем записывать так: $\gamma=\operatorname{Lf}^{p}(\delta)$. Известно, что метрическое пространство $X$ обладает свойством Лефшеца $\operatorname{Lf}^{\infty}$ тогда и только тогда, когда $X$ есть абсолютный окрестностньй экстензор ANE [2]. Менее известен следуюший факт.

ПРЕДЛОЖЕНИЕ 1 [2]. Если $p<\infty$, то метрическое пространство $X$ является локально связным в размерности р тогда и только тогда, когда $X$ обладает свойством Лефшеца в той жсе размерности.

Наконец, приведем обешанньй принцип продолжения Лефшеца. Идея его доказательства фактически имеется в [2, с. 156], поэтому мы его опускаем.

ПрЕдЛОЖЕНИЕ 2 (принцип Лефшеца). Пусть $X \in \mathrm{Lf}^{n}, n \leqslant \infty, u$ задана последовательность $\sigma_{1}, \sigma_{2}, \sigma_{3}, \ldots$ открытых покрытий $X$, для которых каждое последующее $\sigma_{k+1}$ вписано в предыдущее $\sigma_{k}$ (обозначается $\left.\sigma_{k+1} \succ \sigma_{k}\right) u \sigma_{k+1}=\operatorname{Lf}^{n}\left(\sigma_{k}\right)$. Пусть также $(\mathfrak{N}, L)$ есть полиэдр (в слабой топологии), в котором выделень

а) последовательность подполиэдров $\mathfrak{N}=\mathfrak{N}_{1} \supset \mathfrak{N}_{2} \supset \cdots \supset \mathfrak{N}_{m} \supset \cdots$ относительно триангулячии L, для которой открытая звезда $\operatorname{St}_{0}\left(\mathfrak{N}_{m+1}\right)$ лежит ${ }_{\boldsymbol{B}} \mathfrak{N}_{m}$

б) частичное отображсние $\mathfrak{N} \hookleftarrow \mathfrak{N}^{(0)} \stackrel{\varphi}{\rightarrow} X$, заданное на нульмерном остове полиәдра $\mathfrak{N}$, ограничение которого на $\mathfrak{N}_{m}^{(0)}$ является $\sigma_{m+2}$-реализачией.

Тогда существует продолхсение отобрахсения $\varphi$ на $(n+1)$-мерный остов $\widehat{\varphi}$ : $\mathfrak{N}^{(n+1)} \rightarrow X$, для которого ограничение $\widehat{\varphi} \uparrow\left(\mathfrak{N}_{m}^{(n+1)}\right)$ является $\sigma_{m}$-реализачией.

Важность и полезность сформулированного принципа проявляется в соединении с понятиями примькающего покрытия и канонического отображения, как будет видно из дальнейшего. Остановимся на этих понятиях более подробно. 
ОПРЕДЕЛЕНИЕ 3 . Покрытие $\omega \in \operatorname{cov}(Z \backslash A)$ npuмыкaеm к замкнутому подмножеству $A$, если вьполняется одно из двух эквивалентных условий:

1) диаметры элементов $U_{n} \in \omega$ стремятся к 0 всякий раз, как только для некоторой точки $a \in A$ имеет место сходимость расстояний $\rho\left(a, U_{n}\right) \rightarrow 0$;

2 ) для любой точки $a \in A$ и любой ее окрестности $U(a)$ существует окрестность $V(a)$ такая, что звезда множества $V$ относительно покрытия $\omega$, обозначаемая через $\mathrm{N}(V, \omega)=\bigcup\{W \in \omega \mid A \cap W \neq \varnothing\}$, содержится в $U$.

Как показано в [1], [2], примыкающее покрытие всегда существует. Кроме понятия примькающего покрытия чрезвычайно полезны понятия примыкающего отображения и примыкающей последовательности окрестностей.

ОПРЕДЕЛЕниЕ 4. Отображение $\beta: \mathfrak{N}\langle\omega\rangle^{(0)} \rightarrow A$ нульмерного остова $\mathfrak{N}\langle\omega\rangle^{(0)}$ нерва покрытия $\omega$ (понятие нерва $\mathfrak{N}\langle\omega\rangle$ покрытия общеизвестно [1], [2]; симплекс, порожденньй элементами $W_{0}, W_{1}, \ldots, W_{t}$, имеющими непустое пересечение, мы будем обозначать через $\left.\left\langle W_{0}, W_{1}, \ldots, W_{t}\right\rangle\right)$, примькающего к $A$, назьвается примыкаюш, им, если для любой точки $a \in A$ и любой ее окрестности $U(a) \subset Z$ существует окрестность $V(a) \subset Z$ такая, что если $W \cap V(a) \neq \varnothing$ для элемента $W \in \omega$, то $\beta\langle W\rangle \in U(a)$.

Пусть уже заданы примыкающие к $A$ покрытие $\omega$ и отображение $\beta$.

ОПРЕДЕЛЕНИЕ 5. Убывающая последовательность окрестностей $U_{n}$ множества $A$ назьвается примыкающей, если выполняются следующие свойства:

3) $\operatorname{cl}\left(U_{n+1}\right) \subset U_{n}$

4) если $W \in \omega$ и $W \cap U_{n} \neq \varnothing$, то $\operatorname{diam} W<1 / n$ и $\rho(\beta\langle W\rangle, W)<1 / n$.

Фактически в [1], [2] устанавливается существование примыкающих покрытий, отображений и последовательности окрестностей для любого замкнутого подмножества $A$ метрического пространства $Z$. Однако, для доказательства теоремы 3 понадобится более точньй результат.

ПРЕДЛОЖЕНИЕ 3. ДЛя любого замкнутого подмножества А метрического пространства $Z$ существуют примыкающие $\kappa A$ покрытие $\omega \in \operatorname{cov}(Z \backslash A)$, отображсение $\beta: \mathfrak{N}\langle\omega\rangle^{(0)} \rightarrow A$ и последовательность окрестностей $U_{n}$, для которых выполнено свойство:

в) если для последовательности әлементов $W_{n} \in \omega$ точки $\beta\left\langle W_{n}\right\rangle \in A$ являются различными, а последовательность $\left\{\beta\left\langle W_{n}\right\rangle\right\}$ сходится $\kappa$ некоторой точке $а \in A$, то для любой ее окрестности $U_{m} \ni$ а почти все әлементы $W_{n}$ с ней пересекаются $\left(u\right.$, следовательно, $\left.\lim \operatorname{diam} W_{n}=0\right)$.

ДокАЗАТЕЛьство. Пусть для определенности $\operatorname{diam} Z<1$. Зафиксируем последовательность локально конечных покрытий $\omega_{n} \in \operatorname{cov} A$, для которых $\omega_{1}=\{A\}$, каждое последующее покрытие звездно вписано в предыдущее, а калибр cal $\omega_{n}<1 / n$. Выберем подобное продолжение покрытия $\omega_{n}$ до системы $\mu_{n}$ открытых в $Z$ множеств так, чтобы $\mu_{1}=\{Z\}$, каждая последующая система была звездно вписана в предыдущую, а cal $\mu_{n}<1 / 2$. Система $\mu=\left\{\widetilde{H}_{\mu}\right\}$ открытых множеств в $Z$ является подобнылм продолжением покрытия $\omega=\left\{H_{\mu}\right\} \in \operatorname{cov} A$, если

1) $\widetilde{H}_{\mu} \supset H_{\mu}$;

2) из $\cap \widetilde{H}_{\mu_{i}} \neq \varnothing$ следует, что $\cap H_{\mu_{i}} \neq \varnothing$. 
Возможность такого подобного продолжения следует из [2]. Число $\sup \{\operatorname{diam} W \mid W \in \mu\}$ назьвается калибром покрытия $\mu$ и обозначается через саl $\mu$. Не теряя общности, можно считать, что тела $U_{n}$ систем $\mu_{n}$ таковы, что $\operatorname{cl}\left(U_{n+1}\right) \subset U_{n}, \mathrm{a} \cap U_{n}=A$.

В качестве искомого покрытия $\omega \in \operatorname{cov}(Z \backslash A)$ возьмем любое покрытие $\{W\}$, удовлетворяющее следующему свойству:

г) для любого элемента $W \in \omega$ существует число $n$ такое, что $W \subset U_{n-1} \backslash U_{n+1}$, $W \cap U_{n} \neq \varnothing$, и $W \subset S$ для некоторого элемента $S \in \mu_{n-1}$.

Отсюда видно, что $\operatorname{diam} W<1 / n-1$, как только $W \cap U_{n} \neq \varnothing$, и, следовательно, покрытие $\omega$ будет примькающим к $A$.

Каждому элементу $S \in \mu_{n}$ сопоставим точку $a_{S} \in S \cap A \neq \varnothing$. Тогда отображение $\beta: \mathfrak{N}\langle\omega\rangle^{(0)} \rightarrow A$ зададим формулой $\beta\langle W\rangle=a_{S}$, где $W \subset U_{n-1} \backslash U_{n+1}$, a $W \subset S \in \mu_{n}$.

Очевидно, что $\rho(\beta\langle W\rangle, W)<1 /(n-1)$, как только $W \cap U_{n} \neq \varnothing$ для $W \in \omega$, и, следовательно, отображение $\beta$ и последовательность окрестностей $U_{n}$ также будут примыкающими к $A$.

Проверим, что для построенных объектов выполняется свойство а). Предположим противное, т.е. существуют элементы $W_{i} \in \omega$, для которых выполнены одновременно следуюшие соотношения: $W_{i} \subset U_{n_{i}-1} \backslash U_{n_{i}+1} ; W \subset S_{i} \in \mu_{n_{i}-1}$; все точки $\beta\left\langle W_{i}\right\rangle=a_{S_{i}}$ различны, $a_{S_{i}} \rightarrow a \in A$, однако все $n_{i}$ меньше некоторого числа $p$.

Воспользовавшись локальной конечностью покрытий $\omega_{i}$, найдем окрестность $O(a)$, для которой множество $\mathfrak{M}$ элементов $\left\{S \in \mu_{i} \mid i \leqslant p, S \cap O(a) \cap A \neq \varnothing\right\}$ конечно. Так как почти все элементы $S_{i}$ принадлежат этому множеству, то их число конечно. Однако, все точки $a_{S_{i}}$ различны, значит и все элементы $S_{i}$ различны и, следовательно, число различных элементов $S_{i}$ счетно. Противоречие.

Важность и полезность введенных понятий становится очевидной в сочетании с понятием канонического отображения.

ОПРЕДЕЛЕНИЕ 6. Отображение $\theta: Z \rightarrow \mathfrak{N}\langle\omega\rangle$ в нерв покрытия $\omega \in \operatorname{cov} Z$ называется каноническим, если прообраз $\theta^{-1}\left(\mathrm{St}_{0}\langle W\rangle\right)$ открытой звезды вершины $\langle W\rangle$, соответствующей элементу $W \in \omega$, относительно естественной триангуляции полиэдра $\mathfrak{N}\langle\omega\rangle$ лежит в $W$.

Прежде всего отметим, что в случае локально конечных покрытий канонические отображения всег да существуют [1], [2], а существование таких отображений в случае произвольных покрытий легко следует из следующего предложения.

ПРЕДЛОЖЕНИЕ 4. Если симплициальное отображсение $\pi: \mathfrak{N}\langle\sigma\rangle \rightarrow \mathfrak{N}\langle\omega\rangle$ нервов покрытий порождено вписанностью локально конечного покрытия $\sigma \in \operatorname{cov} Z \boldsymbol{\theta}$ покрытие $\omega$, то композииия канонического отображения $\theta: Z \rightarrow \mathfrak{N}\langle\sigma\rangle$ с отображением $\pi$ также есть отображение каноническое.

Сразу же отметим используемое далее свойство канонического отображения $\theta$ :

д) если образ точки $z \in Z$ есть вершина $\langle W\rangle$, то $z \in W$.

Следующее предложение является решающим в доказательстве теоремы 3.

ПРЕДЛОЖЕНИЕ 5. Пусть заданы примыкающие $\kappa$ А покрытие $\omega \in \operatorname{cov}(Z \backslash A)$, отображсение $\beta: \mathfrak{N}\langle\omega\rangle^{(0)} \rightarrow A$, последовательность окрестностей $U_{n}$, удовлетворяющая свойству в) из предложения 3 , а также каноническое отображсние $\theta: Z \backslash A \rightarrow \mathfrak{N}\langle\omega\rangle$. Если подмножество $B \subset Z$ таково, что $\theta(B) \subset \mathfrak{N}\langle\omega\rangle^{(0)}$, то отображение $h: A \cup B \rightarrow A, h\left\lceil A=\operatorname{Id}_{A}, h\lceil B=\beta \circ \theta\right.$, является замкнутым. 
ДокАЗАТЕльство. Предположение о том, что отображение $h$ не является замкнутым влечет существование замкнутого множества $F \subset A \cup B$ и последовательности $f_{n} \in F$, для которой последовательность $\left\{h\left(f_{n}\right)\right\}$, состоящая из различных точек, сходится к некоторой точке $a \in A$, не принадлежащей $h(F)$. Так как $h \uparrow A=\operatorname{Id}_{A}$, можно предполагать, что все $f_{n} \in F \backslash A$ и $h\left(f_{n}\right)=\beta \circ \theta\left(f_{n}\right)$.

Воспользуемся предложением 4 . Тогда для любой окрестности $U_{m}$ почти все элементы $W_{n} \in \omega,\left\langle W_{n}\right\rangle=\theta\left(f_{n}\right)$, с ней пересекаются. В силу определения примькаемости последовательности окрестностей $U_{m}$ следует, что $\rho\left(\beta\left(\theta f_{n}\right), W_{n}\right)<1 / m, \operatorname{diam} W_{n}<1 / m$ для почти всех $n$. В итоге мы имеем $\lim \rho\left(a, W_{n}\right) \rightarrow 0$. Так как $\theta\left(f_{n}\right)=\left\langle W_{n}\right\rangle$, из д) следует, что $f_{n} \in W_{n}$. Поэтому $\lim f_{n}=a$ принадлежит $F$. Следовательно, $a \in F \cap A \subset h(F)$. Получили противоречие.

Теперь вернемся к установлению свойств фильтрации Мориты.

ПрЕдЛОЖЕНИЕ 6. Пусть задана не более чем $(n+1)$-кратная замкнутая сюрбекиия $\eta: W \rightarrow Z$ из нульмерного метрического пространства $W$ на $n$-мерное пространство $Z$. Тогда мнохсество $\Phi_{p}=\left\{z \in Z|| \eta^{-1}(x) \mid \geqslant p\right\}$ является $F_{\sigma}$-мнохсеством размерности $\operatorname{dim} \Phi_{p}=n+1-p$.

ДоказАТЕЛЬСтво. Докажем, что $\operatorname{dim} \Phi_{p}=n+1-p$ и $\Phi_{p}$ есть $F_{\sigma}$-множество. Для этого рассмотрим открыто-замкнутое покрытие $\xi=\left\{H_{\mu}\right\}$ пространства $W$ кратности 1 . Тогда $\zeta=\eta(\xi)$ будет замкнутым локально конечным покрытием $Z$ кратности не более $n+1$. Полагаем $E_{p}(\xi)=\{z \in Z \mid$ кр. $(\zeta) \geqslant p\}$. Легко устанавливается, что $E_{p}(\xi)$ есть замкнутое подмножество в $Z$ и $\operatorname{dim} E_{p}(\xi) \leqslant n+1-p$.

Пусть $\xi_{1}, \xi_{2}, \xi_{3}, \ldots, \xi_{k+1} \succ \xi_{k}$, есть последовательность открыто-замкнутых покрытий $W$ кратности 1 , имеюших $\lim \mathrm{cal} \xi_{n}=0$. Тогда легко проверить, что $\Phi_{p}=\bigcup_{i} E_{p}\left(\xi_{i}\right)$ есть $F_{\sigma}$-множество размерности не более $n+1-p$.

3. Доказательство теоремы 1. Наконец, займемся продолжением частичного отображения $Z \hookleftarrow A \stackrel{\varphi}{\rightarrow} X \in \mathrm{LC}^{p} \cap \mathrm{C}^{q}$. Зафиксируем последовательность $\sigma_{1}, \sigma_{2}, \sigma_{3}, \ldots$ открытых покрытий $X$, для которых $\sigma_{k+1}=\operatorname{Lf}^{p}\left(\sigma_{k}\right), \sigma_{k+1} \succ \sigma_{k}$, а калибр покрытий $\sigma_{k}$ стремится к 0, и согласованно с ней выберем примькающие к $A$ открытое покрытие $\omega \in \operatorname{cov}(Z \backslash A)$, отображение $\beta: \mathfrak{N}\langle\omega\rangle^{(0)} \rightarrow A$ и последовательность окрестностей $U_{n}$.

ПРЕДЛОЖЕНИЕ 7. Существуют примыкающие $\kappa A$ покрытие $\omega \in \operatorname{cov}(Z \backslash A)$, отображсение $\beta: \mathfrak{N}\langle\omega\rangle^{(0)} \rightarrow A$ и последовательность окрестностей $U_{n}$ такие, что последовательность подполиәдров $\mathfrak{N}_{1} \supset \mathfrak{N}_{2} \supset \cdots$ :

$$
\mathfrak{N}_{m}=\bigcup\left\{\left\langle W_{0}, W_{1}, \ldots, W_{t}\right\rangle \mid W_{p} \cap U_{m} \neq \varnothing \text { для всех } p \leqslant t\right\}
$$

и частичное отображсение $\mathfrak{N}_{1} \hookleftarrow \mathfrak{N}_{1}^{(0)} \stackrel{h_{1}}{\rightarrow} X, h_{1}=\varphi \circ\left(\beta \uparrow \mathfrak{N}_{1}^{(0)}\right)$, удовлетворяют свойствам а) и б) критерия Лефиеиа.

ДоказАтЕльство. Дополнительно потребуем от покрытий $\omega_{n} \in \operatorname{cov} A$ и систем $\mu_{n}$, строящихся в доказательстве предложения 3 , такой мелкости, чтобы покрытие $\varphi\left(\omega_{n}\right)$ было вписано в покрытие $\sigma_{n+4}$ для всех $n$. Пусть $\omega, \beta$ и $\left\{U_{n}\right\}$ построены в соответствии с предложением 3.

Для симплекса $\Delta=\left\langle W_{0}, W_{1}, \ldots, W_{t}\right\rangle$ из $\mathfrak{N}_{n}$ справедливы неравенства

$$
\bigcap_{i=0}^{t} W_{i} \neq \varnothing \quad \text { и } \quad W_{p} \cap U_{n} \neq \varnothing
$$


для всех $p \leqslant t$. Это означает, что $W_{p} \subset U_{n-1} \backslash U_{n+2}, W_{p} \subset S_{p} \in \mu_{i}$ для всех $i>n$ и $\beta\left\langle W_{p}\right\rangle \in S_{p} \cap A$. Так как

$$
\bigcap_{i=0}^{t} S_{i} \neq \varnothing, \quad \bigcap_{i=0}^{t} S_{i} \subset S \in \mu_{n-2}
$$

то

$$
\varphi\left(\bigcup_{i=0}^{t}\left(S_{i} \cap A\right)\right) \subset V \in \sigma_{n+2} .
$$

Вложение открытой звезды $\operatorname{St}_{0}\left(\mathfrak{N}_{n+1}\right)$ в $\mathfrak{N}_{n}$ следует из очевидного соображения: если $W_{0} \cap U_{n+1} \neq \varnothing$, а $\bigcap_{i=0}^{t} W_{i} \neq \varnothing$, то $W_{0} \subset U_{n}$ и все $W_{i} \cap U_{n} \neq \varnothing$.

Проверим, что образы вершин симплекса $\Delta$ лежат в элементе покрытия $\sigma_{n+2}$. Для этого заметим, что

$$
h_{1}\left(\cup\left\{\left\langle W_{p}\right\rangle \mid p \leqslant t\right\}\right)=\varphi\left(\cup\left\{\beta\left\langle W_{p}\right\rangle \mid p \leqslant t\right\}\right) \subset \varphi\left(\bigcup_{i=0}^{t}\left(S_{i} \cap A\right)\right) \subset V \in \sigma_{n+2} .
$$

Для частичного отображения $\mathfrak{N}_{1} \hookleftarrow \mathfrak{N}_{1}^{(0)} \stackrel{h_{1}}{\rightarrow} X$ применим принцип продолжения Лефшеца, в силу которого сушествует отображение $g: \mathfrak{N}_{1}^{(p+1)} \rightarrow X$ такое, что его ограничение на $\mathfrak{N}_{n}^{(p+1)}$ дает $\sigma_{n}$-реализацию.

Далее для доказательства разберем наиболее сложньй случай $q \leqslant p$, а затем сведем к нему случай $q>p$. Так как $X \in \mathrm{C}^{q}$, отображение $g$ продолжается до отображения $\widehat{g}: \mathfrak{N}_{1}^{(p+1)} \cup \mathfrak{N}\langle\omega\rangle^{(q+1)} \rightarrow X$ такого, что $\widehat{g}\left\lceil\mathfrak{N}_{1}^{(p+1)}=g, \widehat{g}\left\lceil\mathfrak{N}\langle\omega\rangle^{(0)}=(\varphi \circ \beta)\left\lceil\mathfrak{N}\langle\omega\rangle^{(0)}\right.\right.\right.$.

ПРЕДЛОЖЕНИЕ 8. Пусть $\theta: Z \backslash A \rightarrow \mathfrak{N}\langle\omega\rangle$ есть некоторое каноническое отображение, а $B \subset U_{1} \backslash A$ таково, что $\theta(B) \subset \mathfrak{N}_{1}^{(p+1)}$. Тогда отображение $h: A \cup B \rightarrow X$, $h \uparrow A=\varphi, h\lceil B=\widehat{g} \circ \theta$, является непрерывным.

ДокАЗАТЕЛЬСтво. Достаточно установить непрерьвность $h$ в точке $a \in \operatorname{Bd} A$.

Пусть фиксировано число $\varepsilon>0$ и $\varphi(\mathrm{N}(a, 3 / i) \cap A) \subset \mathrm{N}(\varphi(a), \varepsilon / 2)$. Не теряя общности, можно считать, что из $\mathrm{N}(a, 1 / j) \cap W \neq \varnothing$ следуют неравенства $\operatorname{dim} W<1 / i$ и $\rho(W, \beta\langle W\rangle)<1 / i$ для всех $j \geqslant i$. Тогда если

$$
z \in \bigcap_{i=0}^{m} W_{i} \cap \mathrm{N}\left(a, \frac{1}{j}\right)
$$

a $\theta(z) \in \mathfrak{N}_{1}^{(p+1)}$, то $\rho\left(\beta\left\langle W_{i}\right\rangle, a\right)<1 / j+2 / i \leqslant 3 / i$, а $\theta(z) \in \mathfrak{N}_{i}^{(p+1)}$. В итоге имеем $h(z)=\widehat{g}(\theta(z)) \subset \mathrm{N}\left(\mathrm{N}(\varphi(a), \varepsilon / 2), \sigma_{i+2}\right)$. Так как cal $\sigma_{i} \rightarrow 0$, то $\mathrm{N}\left(\mathrm{N}(\varphi(a), \varepsilon / 2), \sigma_{i+2}\right)$ будет лежать в $\mathrm{N}(\varphi(a), \varepsilon)$.

Вновь рассмотрим резольвенту Мориты $\eta: W \rightarrow Z$. В покрытие $\eta^{-1}(\omega)$ впишем открыто-замкнутое (относительно $W$ ) покрытие $\xi \in \operatorname{cov}\left(\eta^{-1}(Z \backslash A)\right)$ кратности 1. Тогда $\zeta=\eta(\xi)=\left\{W_{\mu}\right\}$ есть локально конечное кратности не более $n+1$ покрытие $Z \backslash A$ из замкнутых в $Z$ множеств, вписанное в $\omega$. При этом введенные ранее множества $E_{p+3}(\xi)$ и $E_{q+3}(\xi)$ будут лежать в $\Phi_{p+3}$ и $\Phi_{q+3}$ соответственно.

$\mathrm{B}$ качестве искомых препятствий к продолжению $\varphi$ возьмем $F_{1}=E_{p+3}(\xi)$ и $F_{2}=$ $E_{q+3}(\xi) \cap\left(Z \backslash U_{1}\right)$. Для них вьполняются требуемые размерностные ограничения и 
условия замкнутости. Убедимся, что $\varphi$ может быть продолжено на дополнение к ним. Для этого в силу предложения 8 достаточно построить каноническое отображение $\theta: Z \backslash A \rightarrow \mathfrak{N}\langle\omega\rangle$ такое, что для $Z_{0}=Z \backslash\left(A \cup F_{1} \cup F_{2}\right)$ имеет место вложение:

e) $\theta\left(Z_{0}\right) \subset \mathfrak{N}_{1}^{(p+1)} \cup \mathfrak{N}\langle\omega\rangle^{(q+1)}$.

Тогда отображение $\widehat{\varphi}: A \cup Z_{0}=Z \backslash\left(F_{1} \cup F_{2}\right) \rightarrow X, \widehat{\varphi} \uparrow A=\varphi, \widehat{\varphi} \uparrow Z_{0}=\widehat{g} \circ \theta$, будет непрерывным отображением, продолжающим $\varphi$. Тем самым, доказательство для случая $q \leqslant p$ будет завершено.

Итак, осушествим намеченное. Для этого рассмотрим ограничение покрытия $\zeta \in$ $\operatorname{cov}(Z \backslash A)$ на открытое в $Z$ подмножество $Z_{0}=Z \backslash\left(A \cup F_{1} \cup F_{2}\right)$. Получающееся покрытие $\vartheta=\left\{H_{\vartheta}\right\} \in \operatorname{cov} Z_{0}$ будет локально конечным и замкнутым. Более того, если $z \in Z_{0} \cap U_{1}$, то кратность покрытия $\vartheta$ в точке $z$, будет не более $p+2$, а если $z \in Z_{0} \backslash U_{1}$, то кратность покрытия $\vartheta$ в точке $z-$ не более $q+2$. Воспользуемся следующим предложением 9 и подобно продолжим покрытие $\vartheta$ до открытого (необязательно локально конечного) покрытия $\widetilde{\vartheta}=\left\{\widetilde{H}_{\vartheta}\right\} \in \operatorname{cov} Z_{0}$. Покрытие $\widetilde{\vartheta}$ можно считать вписанньм в $\omega$ так же, как и $\vartheta \succ \omega$.

ПРЕДЛОЖЕНИЕ 9. Если $\vartheta=\left\{H_{\vartheta}\right\}$ есть локально конечное замкнутое покрытие $Z_{0}$, то существует открытое покрытие $\widetilde{\vartheta}=\left\{\widetilde{H}_{\vartheta}\right\}$ пространства $Z_{0}$ такое, чmo

ж) $\widetilde{H}_{\vartheta} \supset H_{\vartheta}$;

3) $е с л и \cap \widetilde{H}_{\vartheta_{i}} \neq \varnothing$, mo $\cap H_{\vartheta_{i}} \neq \varnothing$

(покрытие $\widetilde{\vartheta}$ назьвается подобным продолэсением покрытия $\vartheta$ ).

ДокАЗАтЕльство. Спектром точки $z \in Z_{0}$ назовем множество $\sigma(z)=\left\{\vartheta \mid z \in H_{\vartheta}\right\}$. Очевидно, что из локальной конечности замкнутого покрытия $\vartheta$ следуют конечность этого множества и существование у любой точки $z \in Z_{0}$ окрестности $U(z)$, удовлетворяющей условию:

и) $\sigma\left(z^{\prime}\right) \subset \sigma(z)$ для всех $z^{\prime} \in U(z)$.

Звездно впишем в покрытие $\varkappa=\left\{U(z) \mid z \in Z_{0}\right\}$ покрытие $\tau=\left\{V(z) \mid z \in Z_{0}\right\}$. Не теряя общности, можно считать, что $V(z) \subset U(z)$ для всех $z \in Z_{0}$. Теперь сопоставим каждой точке $z \in Z_{0}$ точку $f(z) \in Z_{0}$ с условием $\mathrm{N}(V(z), \tau) \subset U(f(z))$. Очевидно, что из

$$
\bigcap_{i=1}^{k} V\left(z_{i}\right) \neq \varnothing
$$

следует

к) $\bigcap_{i=1}^{k} V\left(z_{i}\right) \subset \bigcap_{i=1}^{k} U\left(f\left(z_{i}\right)\right)$, т.е. $\bigcap_{i=1}^{k} \sigma\left(z_{i}\right) \subset \sigma\left(f\left(z_{m}\right)\right)$ для всех $m \leqslant k$.

Положим $\widetilde{H}_{\vartheta}=\mathrm{N}\left(\widetilde{H}_{\vartheta}, \tau\right)$ и установим наиболее сложное свойство з). Пусть

$$
z_{0} \in \bigcap_{i=1}^{k} \widetilde{H}_{\vartheta_{i}}
$$

Тогда существуют элементы $V\left(z_{i}\right) \in \tau, z_{0} \in V\left(z_{i}\right), V\left(z_{i}\right) \cap H_{\vartheta_{i}} \neq \varnothing$. Так как $U\left(z_{i}\right) \cap$ $H_{\vartheta_{i}} \neq \varnothing$, то $\vartheta_{i} \in \sigma\left(z_{i}\right)$, а, стало быть, в силу и), к) имеем $\cap \vartheta_{i} \subset \cup \sigma\left(z_{i}\right) \subset \sigma\left(f\left(z_{m}\right)\right)$ для всех $m \leqslant k$. Тем самым, мы установили, что $f\left(z_{1}\right) \in \cap H_{\vartheta_{i}} \neq \varnothing$. 
Пусть $\theta: Z_{0} \rightarrow \mathfrak{N}\langle\widetilde{\vartheta}\rangle$ является каноническим отображением, а $\pi: \mathfrak{N}\langle\widetilde{\vartheta}\rangle \rightarrow \mathfrak{N}\langle\omega\rangle$-симплициальным отображением, порожденным вписанностью покрытия $\widetilde{\vartheta}$ в покрытие $\omega$. Покажем, что для композиции $\pi \circ \theta: Z_{0} \rightarrow \mathfrak{N}\langle\omega\rangle$, которая также является каноническим отображением, справедливо вложение е).

В самом деле, пусть

$$
z \in \bigcap_{i \leqslant k} \widetilde{H}_{\vartheta_{i}},
$$

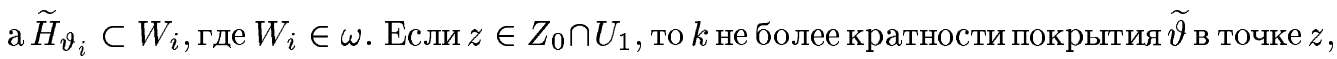
которая в свою очередь будет не более $p+2$. Кроме того, симплекс $\Delta=\left\langle W_{0}, \ldots, W_{k}\right\rangle \in$ $\mathfrak{N}_{1}^{(p+1)}$, так как $z \in \widetilde{H}_{\vartheta_{i}} \cap U_{1} \subset W_{i} \cap V_{1} \neq \varnothing$ для всех $i \leqslant k$. В итоге, $\pi \circ \theta(z) \in \mathfrak{N}_{1}^{(p+1)}$.

Если же $z \in Z_{0} \backslash U_{1}$, то $k$ не более кратности покрытия $\widetilde{\vartheta}$ в точке $z$, которая в свою очередь будет не более $q+2$. Следовательно, $\pi \circ \theta(z) \in \mathfrak{N}\langle\omega\rangle^{(q+1)}$. Тем самым, случай $q \leqslant p$ полностью разобран.

Пусть теперь $q>p$. Так как $X \in \mathrm{LC}^{p} \cap \mathrm{C}^{p}$, то в силу уже доказанного существуют препятствия $F_{1}^{\prime} \subset \Phi_{p+3}$ и $F_{2}^{\prime} \subset \Phi_{p+3}$ для продолжения частичного отображения $\varphi$. В качестве первого препятствия $F_{1} \subset \Phi_{p+3}$ возьмем $F_{1}^{\prime} \cup F_{2}^{\prime}$, в качестве второго препятствия $F_{2} \subset \Phi_{q+3}$ возьмем $\varnothing$.

\section{СПИСОК ЦИТИРОВАННОЙ ЛИТЕРАТУРЫ}

[1] Борсук К. Теория ретрактов. М.: Наука, 1971.

[2] Hu S. T. Theory of retracts. Detroit: Wayne State Univ. Press, 1965.

[3] Eilenberg S. Un théorème de dualité // Fund. Math. 1936. V. 26. P. 280-282.

[4] Borsuk K. Un théorème sur les prolongements des transformations // Fund. Math. 1937. V. 29. P. 161-166.

[5] Богатый С. А. О метрических ретрактах // Докл. АН СССР. 1972. Т. 204. № 3. С. 522-524.

[6] Левшенко Б. Т. Размерность метрических пространств и ретракция // Fund. Math. 1969. V. 66. P. 1-5.

[7] Никифоров В.А. Продолжение селекции к многозначному отображению и теорема двойственности Эйленберга-Борсука // Вестн. МГУ. Сер. 1. Матем., мех. 1987. №6. С. 57-59.

[8] Engelking P. Closed mapping on complete metric spaces // Fund. Math. 1971. V. 70. P. 103-107.

[9] Александров П. С., Пасынков Б. А. Введение в теорию размерности. М.: Наука, 1974.

[10] Федорчук В. В., Филиппов В. В. Общая топология. Основные конструкции. М.: Изд-во МГУ, 1988.

[11] Michael E. Continuous selection. II // Ann. Math. (2). 1956. V. 64. № 3. P. 562-580.

[12] Дранишников А.Н. Теорема Эйленберга-Борсука для отображений в произвольный комплекс // Матем. сб. 1994. Т. 184. №4. С. 82-90.

Брестский государственный педагогический институт им. А. С. Пушкина Московский государственный университет им. М.В. Ломоносова

E-mail: box@univer.belpak.brest.by

Поступило 16.02 .95

Исправленный вариант 22.08.97 